\title{
Evaluation Measures for Event Detection Techniques on Twitter Data Streams
}

\author{
Andreas Weiler ${ }^{(凶)}$, Michael Grossniklaus, and Marc H. Scholl \\ Department of Computer and Information Science, University of Konstanz, \\ P.O. Box 188, 78457 Konstanz, Germany \\ \{andreas.weiler,michael.grossniklaus, marc.scholl\}@uni-konstanz.de
}

\begin{abstract}
Twitter's popularity as a source of up-to-date news and information is constantly increasing. In response to this trend, numerous event detection techniques have been proposed to cope with the rate and volume of social media data streams. Although most of these works conduct some evaluation of the proposed technique, a comparative study is often omitted. In this paper, we present a series of measures that we designed to support the quantitative and qualitative comparison of event detection techniques. In order to demonstrate the effectiveness of these measures, we apply them to state-of-the-art event detection techniques as well as baseline approaches using real-world Twitter streaming data.
\end{abstract}

\section{Introduction}

Microblogging is a form of social media that enables users to broadcast short messages, links, and audiovisual content to a network of followers as well as to their own public timeline. In the case of Twitter, the most popular and fastest-growing microblogging service, these so-called tweets can contain up to 140 characters. Twitter's 288 million monthly active users produce a total of over 500 million tweets per day ${ }^{1}$. As a consequence, several proposals have been made to leverage Twitter as a source of up-to-date news and information, e.g., to respond to natural disasters [13], to track epidemics [7], or to follow political elections [17].

A number of techniques have been designed and developed to detect such events in the Twitter social media data stream. Typically, they adopt the definition of an event introduced by research on Topic Detection and Tracking (TDT), i.e., a real-world occurrence that takes place in a certain geographical location and over a certain time period [2]. The main focus of these event detection techniques lies in addressing the specific requirements introduced by Twitter data, such as the brevity of tweets together with the fact that they contain a substantial amount of spam, typos, slang, etc. Although most proposals provide some qualitative evidence to motivate the benefits of the technique, few perform a quantitative evaluation or compare their results to competing approaches.

We argue that this lack of comparative evaluation is explained by the fact that measuring the quantitative and qualitative performance of event detection

\footnotetext{
$\overline{{ }^{1} \text { https://about.twitter.com/company/. }}$
} 
techniques for Twitter data is itself a challenging research question. Crafting a gold standard manually in order to use textbook precision and recall measures is painstakingly slow and does therefore not scale to the volumes of data generated by Twitter users. In order to address this requirement, we build on our previous work in this field [15] and, in this paper, propose several scalable measures that can be automatically applied to the results of current and future event detection techniques. The specific contributions of this paper are as follows.

1. Definition of evaluation measures to automatically evaluate the precision and recall of event detection techniques for Twitter (Sect.3).

2. Realization of several state-of-the-art event detection techniques as query plans for a data stream management (Sect. 4).

3. Detailed study using real-life Twitter data that demonstrates the ability of our measures to evaluate the different techniques (Sect. 5).

As our evaluation approach is platform-based and modular, it will also enable further systematic performance studies of future event detection techniques.

\section{Background}

Several event detection techniques for Twitter data streams have recently been proposed. Farzindar and Khreich [8] survey sixteen techniques and conclude that most approaches are evaluated by self-defined measures with manually labeled reference data sets. Also, almost none of the reviewed techniques are compared to competing approaches. In the following, we summarize what evaluations have been performed by the authors of the most-cited approaches and what corpora are currently available for evaluation purposes. Our findings show that neither the works discussed below nor the sixteen in the above-mentioned survey provide a general solution that can be used to evaluate approaches comparatively.

\subsection{Evaluation of Event Detection Approaches}

enBlogue [3] identifies unusual shifts in the co-occurrence of tag pairs and reports these shifts as events, which are rated in terms of quality in a user study. TwitterMonitor [9] detects "bursty" keywords and then groups them into trends, which are visualized in order for users to decide whether a trend is interesting or not. Cordeiro [6] proposes the use of continuous wavelet analysis to detect event peaks in a signal based on hashtags frequency and summarizes the detected events into topic clusters with latent dirichlet allocation (LDA [5]). The technique is evaluated using a visualization of the results obtained from an eight day dataset with 13.6 million tweets. All of these manual evaluations are, however, not general in the sense that they do not scale and might suffer from human error or bias. Weng et al. [17] present a technique that uses term frequencies of individual terms as a signal for discrete wavelet analysis to detect event terms. Then, graph partitioning is used to group similar terms into events. The approach is evaluated using a custom ground truth that is built using LDA on a dataset containing of 4,331,937 
tweets collected from Singapore-based users. After cleaning and filtering, a total of 8,140 unique words are retained per month of Twitter data. Detected events are compared to this ground truth on a daily basis. The result of this evaluation is that detected events are plausible, but also that there are several days with no events detected. Since event detection is often time-critical and events should be reported in (near) real-time, this coarse evaluation technique is not suited for general evaluations.

\subsection{Available Corpora for Evaluation}

In our work, we propose to address the problem of comparing various event detection techniques by defining general evaluation measures. In contrast to our approach, which does not rely on an existing reference data set, other works focus on the creation of evaluation corpora for Twitter-related analysis techniques.

For example, McCreadie et al. [10] created a set of approximately 16 million tweets for a two-week period. The proposed corpus contains an average of 50,000 tweets per hour, which is almost equal to the number of tweets per minute in our $10 \%$ stream. Since no language filtering is performed, which can be estimated to retain approximately $30 \%$ (see Fig. 2) of these tweets, only about 4,800,000 tweets are in English. Furthermore, their list of 49 reference topics for the twoweeks period is very limited and no description is given how these topics were created. Finally, this corpus focusses on ad-hoc retrieval tasks and is, therefore, not suited for the large-scale evaluation of event detection approaches. Becker et al. [4] created a Twitter corpus, that consists of over 2,600,000 Twitter message posted during February 2010. Since they only used their own approach to detect events, the corpus is strongly biased to their technique and not suited for general evaluation purposes. Furthermore, no list of reference events is provided and the data set is restricted to tweets from users located in New York City. Petrović et al. [12] presented a corpus of 50 million tweets, created from a manual analysis of the Twitter data stream between July 2011 and mid-September 2011, which led to the definition of 27 events for the whole time-frame. This very low number of "hits" makes it difficult to compare different event detection methods, if techniques used are very different. McMinn et al. [11] propose a methodology to create a corpus for evaluating event detection approaches. They used existing event detection techniques $[1,12]$ together with Wikipedia to build a set of candidate events and associated tweets. The final corpus covers four weeks with about 120 million tweets and more than 500 events. However, since events are given in prose, they cannot be compared automatically to results of event detection techniques. It is important to note that all of these corpora only consist of tweet identifiers. To use them, the tweets themselves have to be crawled, which can be time-consuming and error-prone as some tweets might not exist anymore.

\section{Measures}

In order to address the lack of a common evaluation method for event detection in Twitter data streams, we propose a number of measures. Our goal is to define 
measures that can easily be used by other researchers and that do not deprecate over time as most reference corpora do. While all of our measures support relative comparisons, we do not claim that they can be used to draw absolute conclusions. A single event detection technique can, therefore, only be evaluated "against itself", e.g., with respect to different parameter settings or to confirm that improvements to the technique yield better results. For a set of techniques, the measures can be used to rank them with respect to different criteria. In this paper, we focus on the second application.

Run-Time Performance. We measure run-time performance as the number of tweets that an approach processes per second. This measure is important to judge the feasibility of a technique. Most event detection techniques can be configured based on numerous parameter that influence both the processing speed and result quality. In combination with other measures, the run-time performance measure can, therefore, also be used to study the trade-off between these two objectives.

Duplicate Event Detection Rate (DEDR). This measure captures the percentage of duplicate events detected by an approach. The implementations of state-of-the-art event detection techniques used in this paper avoid the reporting of duplicate events within their processing time-frame, e.g., a one-hour window. Nevertheless, important or long-lasting events can reoccur across several timeframes and, therefore, expecting a $0 \%$ rate of duplicate events is not reasonable.

Precision. Our precision measure is composed of two components. First, we query Google using the five event terms and a specific date range as search query input. Doing so, we are able to verify if the detected event has been described by an important article returned by Google for the corresponding time frame. As important articles we define search results that are from one of the top 15 news websites such as CNN, CBSNews, USAToday, BBC, and Reuters. For the second part of our precision measure, we query the archive of the New York Times $^{2}$ with the five event terms as well as the specific date range. Since the number of hits $(h)$, which are in the range between 0 and 10 both for Google $\left(h^{\mathrm{G}}\right)$ or New York Times $\left(h^{\mathrm{NYT}}\right)$, is an indicator of how important a reported event is, we calculate the final precision score for all results $(N)$ by weighting the single results as

$$
\frac{1}{N} \sum_{i=0}^{N}\left(\frac{1}{2} h_{i}^{\mathrm{G}}+\frac{1}{2} h_{i}^{\mathrm{NYT}}\right) .
$$

Recall. We propose to calculate recall by crawling the world news headlines on the Reuters website ${ }^{3}$ for the days corresponding to the analysis. Each headline is represented as a list of terms $T^{\mathrm{hl}}$. With this measure we intend to reflect the percentage of detected events with respect to important news appearing on a real-world news archive. To weigh the single results, we check for each term in a news headline, which reported event, represented as a list of terms $T^{\mathrm{e}}$, has

2 http://query.nytimes.com/search/sitesearch/.

3 http://www.reuters.com/news/archive/worldNews?date=02112015/. 
the maximal similarity value (max_sim). Since we exclude matches on one term only, this similarity value can either be two, three, four, or five terms. With this weighting, we calculate the final recall score for all headlines $(\mathrm{N})$ as

$$
\frac{1}{N} \sum_{i=0}^{N} \frac{1}{2} \max \_\operatorname{sim}\left(T_{i}^{\mathrm{hl}}, T^{\mathrm{e}}\right) .
$$

\section{Event Detection Approaches}

In order to realize streaming implementations of state-of-the-art event detection techniques for Twitter, we use Niagarino ${ }^{4}$, a data stream management system developed and maintained by our research group. The main purpose of Niagarino is to serve as an easy-to-use and extensible research platform for streaming applications such as the ones presented in the paper. Based on its operator-based processing pipeline our implementations are modular and can be easily configured. For example, we can configure the approaches to report the same number of events, which are represented as one main event term together with four associated event description terms. Using a common implementation platform also has the advantage that run-time performance results can be compared fairly.

For the evaluation of our measures we take nine different approaches into account. Figure 1 shows the Niagarino-based implementations of these approaches. Additionally, the pre-processing pipeline, which is used by all approaches, is shown on the left. The pre-processing removes all non-English tweets and retweets. Then, it tokenizes and unnests the terms of the remaining tweets. It also discards terms that can be classified as stop-words or as noise (e.g., too short, invalid characters, etc.). Finally, a tumbling window of size $s_{\text {input }}$ is continuously applied and its contents are forwarded to the subsequent operators.

At the bottom of Fig. 1, the query plans for $L D A$, TopN, LastN, RandomEvents (RE), and FullRandom (FR) are shown. Since these approaches are not specifically tailored to the task of event detection, we use them as baseline approaches in order to confirm that the proposed measures are discriminating. $L D A$ [5] uses the probabilities of terms in documents and groups those terms together that have the highest probability of belonging together. We realized LDA in Niagarino based on its user-defined function operator. Since LDA is normally used for topic modeling, we equate a topic to an event. The parameters that can be set for this approach include the number of topics, the number of terms per topic, and the number of iterations of the probability modeling. As there are a lot of repeating terms in tweets and also per time window, we expect that this technique is not suitable for event detection and therefore classify it as a baseline method. The other four baseline techniques use a grouping operator followed by a selection operator. FR constructs "events" by randomly selecting five terms from all distinct terms in a time window. $R E$ selects the main event term in the same way as $F R$, but uses the four most co-occurring terms of the

\footnotetext{
${ }^{4}$ http://www.informatik.uni-konstanz.de/grossniklaus/software/niagarino/.
} 


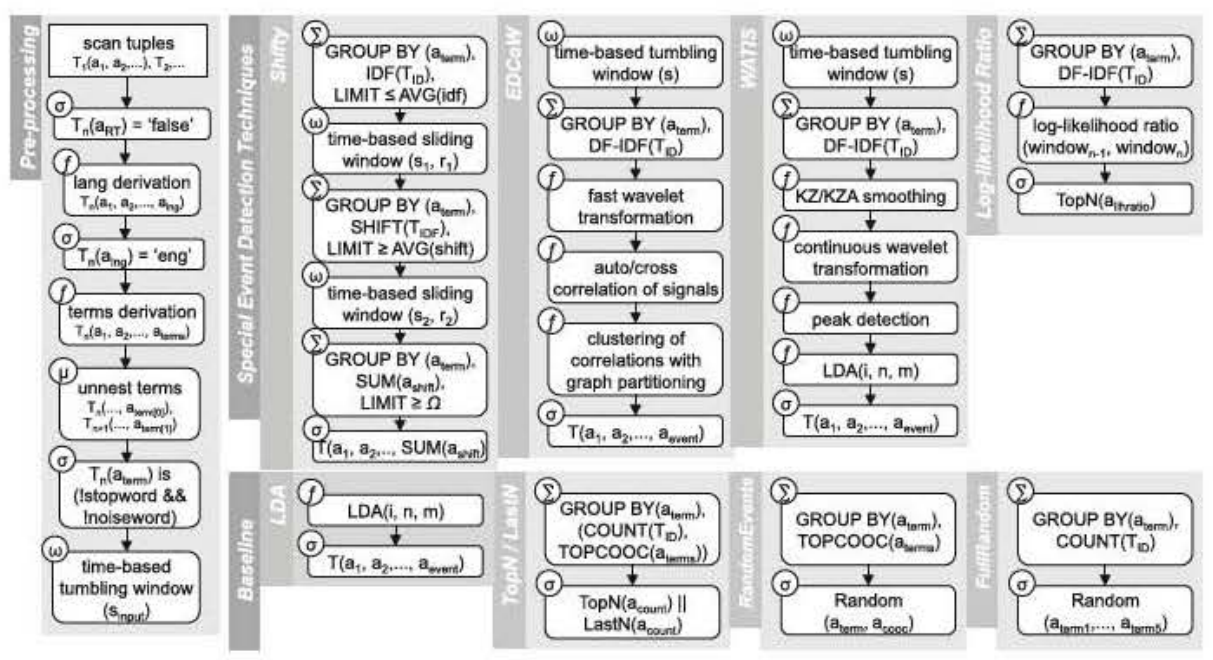

Fig. 1. Niagarino query plans of the studied event detection techniques.

event term as the associated event description terms. Both of these approaches report $N$ events per time window. The next two approaches, TopN and LastN are based on the IDF score of single terms among all distinct terms in the time window. While Top $N$ selects the $N$ most frequent terms, Last $N$ selects the $N$ terms with the lowest frequency. Both of them report the selected event terms together with the four most co-occurring terms.

In addition to these baseline approaches, we implemented several techniques that have been proposed to detect events in Twitter data streams. The corresponding Niagarino query plans are shown at the top of Fig. 1. The first technique, $L L H$, is a reimplementation of Weiler et al. [16], which is realized as a log-likelihood ratio user-defined function that is applied to the grouped set of terms of a time window. In contrast to the original technique that detected events for pre-defined geographical areas, we adjusted the approach to calculate the log-likelihood measure for the frequency of all distinct terms in the current time window against their frequency in the past time windows. Events are reported by selecting the top $N$ terms with the highest log-likelihood ratio together with the corresponding top four most co-occurring terms. Since, these are the terms with the highest abnormal behavior in their current frequency with respect to their historical frequency, we define these terms to be events. The second technique, Shifty, is a reimplementation of Weiler et al. [14]. In contrast to the original paper, which additionally analysis bigrams, we now only use single terms in the analysis. The technique calculates a measure that is based on the shift of IDF values of single terms in pairs of successive sliding windows of a pre-defined size $s_{\text {input }}$. First, the IDF value of each term in a single window is continuously computed and compared to the average IDF value of all terms within that window. Terms with an IDF value above the average are filtered out. The next step builds a window with size $s_{1}$ that slides with range $r_{1}$ in order 
to calculate the shift from one window to the next. In this step, the shift value is again checked against the average shift of all terms and only terms with a shift above the average are retained. In the last step, a new sliding window with size $s_{2}$ that slides with range $r_{2}$ is created. The total shift value is computed as the sum of all shift values of the sub-windows of this window. If this total shift value is greater than the pre-defined threshold $\Omega$, the term is detected as event and reported together with its top four co-occurrence terms. The third technique, WATIS, is an implementation of Cordeiro [6]. The algorithm partitions the stream into intervals of $s$ seconds and builds DF-IDF signals for each distinct term. Due to the noisy nature of the Twitter data stream, signals are then processed by applying an adaptive Kolmogorov-Zurbenko filter (KZA), a low-pass filter that smoothens the signal by calculating a moving average with $i_{\text {kza }}$ iterations over $N$ intervals. It then uses a continuous wavelet transformation to construct a time/frequency representation of the signal and two wavelet analyses, the tree map of the continuous wavelet extrema and the local maxima detection, to detect abrupt increases in the frequency of a term. In order to enrich events with more information, the previously mentioned LDA algorithm (with $i_{\mathrm{LDA}}$ iterations) is used to model one topic consisting of five terms. After the LDA phase the event is reported. Finally, the fourth technique, EDCo $W$, is an implementation of Weng et al. [17]. The first step of the algorithm is to partition the stream into intervals of $s$ seconds and to build DF-IDF signals for each distinct term in the interval. These signals are further analyzed using a discrete wavelet analysis that builds a second signal for the individual terms. Each data point of this second signal summarizes a sequence of values from the first signal with length $\Delta$. The next step then filters out trivial terms by checking the corresponding signal auto-correlations against a threshold $\gamma$. The remaining terms are then clustered to form events with a modularity-based graph partitioning technique. Insignificant events are filtered out using a threshold parameter $\epsilon$. Since this approach detects events with a minimum of two terms, we introduced an additional enrichment step that adds the top co-occurring terms to obtain events with at least five terms. Since the original paper fails to mention the type of wavelet that was used, we experimented with several types. The results reported in this paper are based on the Discrete Meyer wavelet.

\section{Evaluation}

In order to demonstrate that the measures proposed in this paper are discriminating, we run experiments against three different real-world Twitter stream datasets (consisting of five days each) that we collected. The three datasets respectively contain the days of February 1 to 6,11 to 16 , and 21 to 26 , 2015 (EST). By using the Gardenhose access of the Twitter streaming API, we are able to obtain a randomly sampled $10 \%$ stream of all public tweets. The collection contains an average of 2.2 million tweets per hour and almost 50 million tweets per day. We pre-filtered the dataset for tweets with English language content by using a pre-existing Java library ${ }^{5}$. After this step, the dataset

\footnotetext{
${ }^{5}$ https://code.google.com/p/language-detection/.
} 


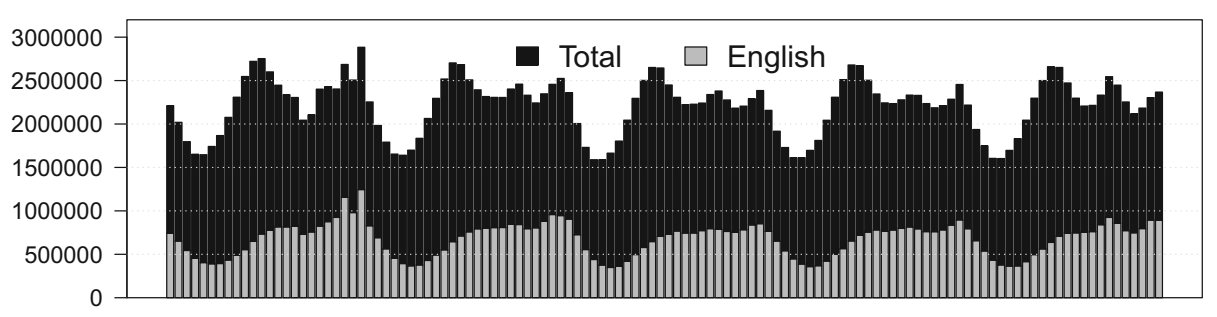

Fig. 2. Average hourly total and English tweets for all three datasets.

contains an average of 660,000 tweets per hour and 16 million tweets per day. Figure 2 shows the distribution of the total and the English number of tweets per hour for each day as an average of all three datasets.

\subsection{Experimental Setup}

The event detection techniques that we use for our evaluation have all been defined with slightly different use cases in mind. In order to fairly compare them, we defined a common task that all of the techniques can accomplish. As we are interested in (near) real-time event detection, we set the length of the time-window used for event reporting to one hour. This means that after each hour of processing the techniques need to report the results obtained so far. Note that within the time window of one hour no duplicate events are possible for any technique. As the number of events reported by the different techniques may vary significantly (depending on the parameter settings), we adjusted the parameters of each technique to report a number of events in a certain range. For techniques, for which the number of detected events is based on a single parameter $N$, we set this parameter to obtain 15 events per hour, which results in 1800 events per dataset. Note that some techniques report a few events with less than five terms, which are discarded. We compensated for this behavior by adjusting the parameters of such event detection techniques accordingly. Table 1 summarizes the parameter settings used. Note, however, that these settings are purely required to obtain comparable output and might not correspond to the optimal settings for each techniques. Also, it is unlikely that events are uniformly distributed over the hours of a day. Using these setting, we obtain 1,745 events for Shifty, 1,520 for WATIS, and 2,020 for EDCoW.

Table 1. Parameter settings for Shifty, WATIS, and EDCoW.

\begin{tabular}{l|l}
\hline Approach & Parameters \\
\hline Shifty & $s_{\text {input }}=1 \mathrm{~min}, s_{1}=2 \mathrm{~min}, r_{1}=1 \mathrm{~min}, s_{2}=4 \mathrm{~min}, r_{2}=1 \mathrm{~min}, \Omega=30$ \\
\hline WATIS & $s=85 \mathrm{~s}, N=5$ intervals, $i_{\mathrm{kza}}=5, i_{\mathrm{lda}}=500$ \\
\hline EDCoW & $s=10 \mathrm{~s}, N=32$ intervals, $\gamma=1, \epsilon=0.2$ \\
\hline
\end{tabular}




\section{$5.2 \quad$ Results}

In the following, we present the results of our evaluation. Note, that we summarized the results of both datasets as an average. First, we start with the run-time performance. Run-time performance was measured using Oracle Java 1.8.0_25 (64 bit) on server-grade hardware with 2 Intel Xeon E5345s processors at $2.33 \mathrm{GHz}$ with 4 cores each and $24 \mathrm{~GB}$ of main memory.

Figure 3 shows the run-time performance results for all techniques measured in terms of average throughput (tweets/ second) for all three datasets. The baseline techniques, except for LDA, as well as the $L L H$ technique achieve the highest throughput with around 12,000 tweets/second. The rate of our Shifty technique is lower at around 8,000 tweets/second. How-

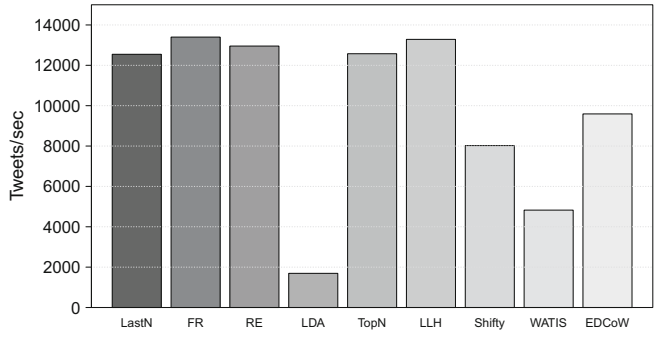

Fig. 3. Run-time performance. ever, it should be noted that Shifty is the only online technique that processes the input incrementally. Therefore, Shifty's performance does not depend on changes to the reporting schedule that we used (after each hour), which will affect the throughput of all other approaches. In contrast to WATIS, EDCoW scores very well. Since WATIS uses $L D A$ at the end of processing to create the final events, this result is not surprising. As we see, applying $L D A$ with 500 iterations is the slowest approach with around 1,700 tweets/second. If we take into account the 50 million tweets per day ( $\sim 580$ per second) of the $10 \%$ stream, we can observe that all techniques could process this stream in (near) real-time and are therefore feasible. However, if these techniques were applied to the full $100 \%$ stream ( 5, 800 tweets per second), WATIS and $L D A$ would not be feasible. Based on these observations, we conclude that our measure for run-time performance is discriminating and can be used to judge the feasibility of approaches.

In contrast to run-time performance, the remaining three measures assess the task-based performance, i.e., the quality of an event detection technique. To further evaluate our measures, we also include the results of applying them to the so-called Trending Topics (TT) of Twitter in the following discussion. We collected the top 15 trending topics and enriched them by querying the Twitter API for the most current tweets belonging to each topic. The enrichment process also tokenizes and cleans the obtained tweets, and summarizes the five most cooccurring terms to a final event. Hereby, we also get 1,800 events per dataset. We begin by presenting the results obtained from our DEDR measure. For each technique, we calculate the percentage of events, which are classified as duplicates. As this classification is configurable, we present results obtained by requiring that one, two, three, four, or all five event terms need to be equal (DEDR1,..., $D E D R 5$ ). Figure 4 plots the average results of the duplicate event detection rate for all datasets. We can observe that all techniques report a very high number 


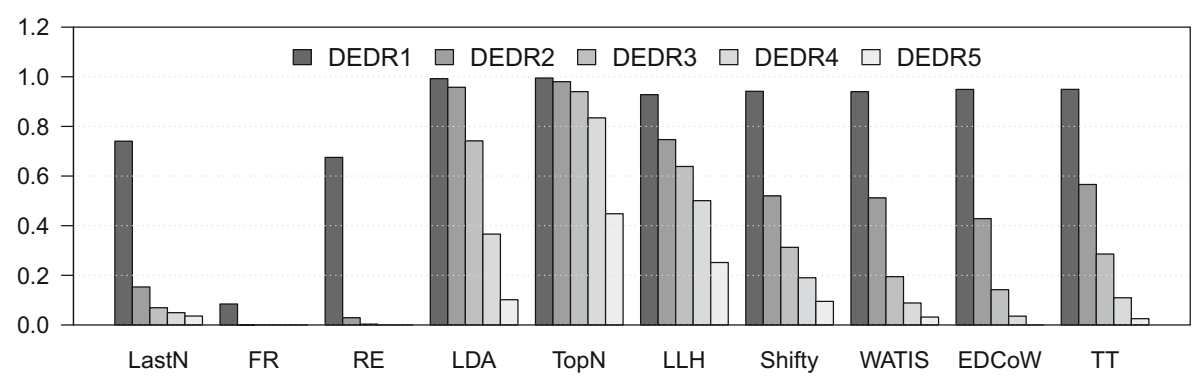

Fig. 4. Average duplicate event detection rate.

of duplicates for $D E D R 1$. Since the terms of $F R$ and $R E$ are randomly chosen, they generally report a lower number of duplicates. From the event detection techniques, the results for Shifty, WATIS, and EDCoW closely resemble the results of applying our $D E D R$ measure to $T T$, whereas the all other approaches have significantly different profiles. We therefore argue that $D E D R$ is a useful measure to characterize event detection techniques.

For the evaluation of our precision and recall measures, we only use events that were not filtered out by $D E D R 3$, i.e., all events with three or more common terms are removed from the result set and only the remaining non-duplicate events are further analyzed. Note that this results in an implicit inclusion of the $D E D R$ measure in our precision and recall measures. Figure 5 shows the average precision, recall, and F-measure over all three data sets for all techniques. Based on these measure, we observe that all of the dedicated event detection techniques clearly outperform the baseline approaches. This finding confirms the validity of the precision and recall measure proposed in this paper. We conclude our evaluation by discussing the results shown in Fig. 5 in more detail. First, we note that the scores are generally very low. However, since we are only interested in relative comparisons, this is not a problem. Among the baseline approaches, both $L D A$ and $R E$ score comparable to dedicated event detection techniques with respect to specific measures. The precision of $L D A$ is higher than the one of $L L H$ and Shifty, RE scores well in terms of recall. In both cases, this result can be explained with the way these approaches work. Also, it demonstrates the importance of studying both precision and recall, which we support with our F-measure. The best approaches according to our measures are the advanced WATIS and EDCo $W$ techniques, which are also the most cited event detection techniques. Since $E D C o W$ produces the most events of all techniques, its parameters could also be adjusted to increase its precision score. Also, the basic enrichment process that we implemented for EDCo $W$ could be improved. For example, WATIS uses $L D A$ for the same purpose and scores very well in terms of recall. Our own techniques, $L L H$ and Shifty, do not perform as well as the two advanced techniques. However, we note that Shifty is the only online event reporting technique and therefore only uses very short time intervals (of four minutes in this case) instead of a full hour to classify terms as events. 


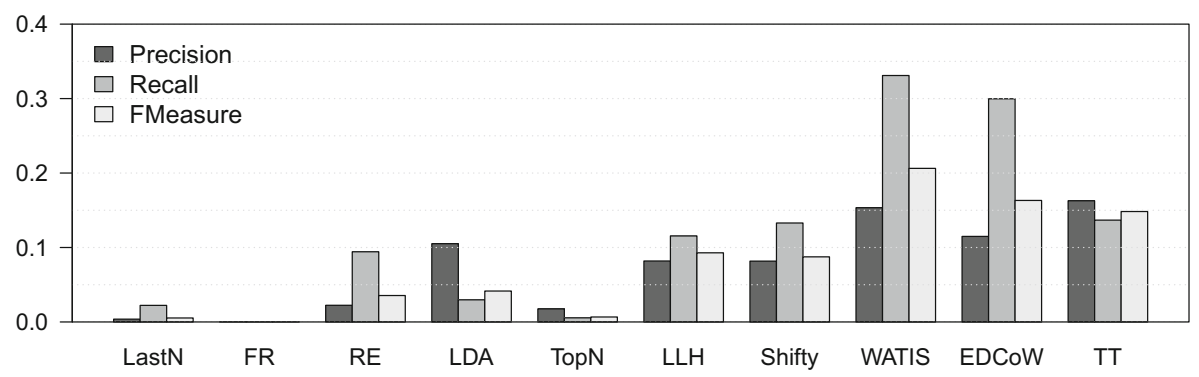

Fig. 5. Precision, Recall, and F-Measure of all techniques.

Additionally, we do not use bigrams in this paper as opposed to the original Shifty algorithm. $L L H$ was originally designed to use both the spatial and the time dimension to detect unusual rates of terms in pre-defined geographical areas over time. In this paper, we only use the time dimension, which has weakened the performance of the approach. Finally, our measures assign high precision and recall scores to the Twitter Trending Topics $(T T)$. However, in contrast to our results, TT is based on the full $100 \%$ stream.

\section{Conclusions}

In this paper, we have addressed the lack of quantitative and comparative evaluation of event detection techniques by proposing a number of measures, both for run-time and task-based performance. In contrast to previous evaluation methods, all our measures can be automatically applied to evaluate large results sets without the requirement of an existing gold standard. In order to demonstrate the validity of our proposed measures, we have studied them based on several baseline approaches and state-of-the-art event detection techniques. We have shown that our measures are able to discriminate between different techniques and support relative comparisons.

As future work, we plan to further confirm the findings presented in this paper by implementing additional event detection techniques, such as enBlogue [3], in our evaluation framework. Based on these fully validated measures, we will tune the parameters of each technique, which will enable us to draw absolute conclusions about their performance.

Acknowledgement. We would like to thank our students Christina Papavasileiou and Harry Schilling for their contributions to the implementations of WATIS and EDCoW.

\section{References}

1. Aggarwal, C.C., Subbian, K.: Event detection in social streams. In: Proceedings of the SIAM International Conference on Data Mining (SDM), pp. 624-635 (2012) 
2. Allan, J.: Topic Detection and Tracking: Event-based Information Organization. Kluwer Academic Publishers, The Netherlands (2002)

3. Alvanaki, F., Michel, S., Ramamritham, K., Weikum, G.: See what's enBlogue: real-time emergent topic identification in social media. In: Proceedings of the International Conference on Extending Database Technology (EDBT), pp. 336-347 (2012)

4. Becker, H., Naaman, M., Gravano, L.: Beyond trending topics: real-world event identification on twitter. In: Proceedings of the International Conference on Weblogs and Social Media (ICWSM), pp. 438-441 (2011)

5. Blei, D.M., Ng, A.Y., Jordan, M.I.: Latent dirichlet allocation. J. Mach. Learn. Res. 3, 993-1022 (2003)

6. Cordeiro, M.: Twitter event detection: combining wavelet analysis and topic inference summarization. In: Proceedings of the Doctoral Symposium on Informatics Engineering (DSIE) (2012)

7. Culotta, A.: Towards detecting influenza epidemics by analyzing twitter messages. In: Proceedings of the Workshop on Social Media Analytics (SOMA), pp. 115-122 (2010)

8. Farzindar, A., Khreich, W.: A survey of techniques for event detection in twitter. Comput. Intell. 31(1), 132-164 (2015)

9. Mathioudakis, M., Koudas, N.: TwitterMonitor: trend detection over the twitter stream. In: Proceedings of the International Conference on Management of Data (SIGMOD), pp. 1155-1158 (2010)

10. McCreadie, R., Soboroff, I., Lin, J., Macdonald, C., Ounis, I., McCullough, D.: On building a reusable twitter corpus. In: Proceedings of the International Conference on Research and Development in Information Retrieval (SIGIR), pp. 1113-1114 (2012)

11. McMinn, A.J., Moshfeghi, Y., Jose, J.M.: Building a large-scale corpus for evaluating event detection on twitter. In: Proceedings of the International Conference on Information and Knowledge Management (CIKM), pp. 409-418 (2013)

12. Petrović, S., Osborne, M., Lavrenko, V.: Using paraphrases for improving first story detection in news and twitter. In: Proceedings of the Conference of the North American Chapter of the Association for Computational Linguistics: Human Language Technologies (NAACL HLT), pp. 338-346 (2012)

13. Sakaki, T., Okazaki, M., Matsuo, Y.: Earthquake shakes twitter users: real-time event detection by social sensors. In: Proceedings of the International Conference on World Wide Web (WWW), pp. 851-860 (2010)

14. Weiler, A., Grossniklaus, M., Scholl, M.H.: Event identification and tracking in social media streaming data. In: Proceedings of the EDBT Workshop on Multimodal Social Data Management (MSDM), pp. 282-287 (2014)

15. Weiler, A., Grossniklaus, M., Scholl, M.H.: Run-time and task-based performance of event detection techniques for twitter. In: Zdravkovic, J., Kirikova, M., Johannesson, P. (eds.) CAiSE 2015. LNCS, vol. 9097, pp. 35-49. Springer, Heidelberg (2015)

16. Weiler, A., Scholl, M.H., Wanner, F., Rohrdantz, C.: Event identification for local areas using social media streaming data. In: Proceedings of the SIGMOD Workshop on Databases and Social Networks (DBSocial), pp. 1-6 (2013)

17. Weng, J., Lee, B.S.: Event detection in twitter. In: Proceedings of the International Conference on Weblogs and Social Media (ICWSM), pp. 401-408 (2011) 